\title{
Power Penalties Due to Brillouin and Rayleigh Scattering in a Bidirectional Coherent Transmission System
}

\author{
M. Oskar van Deventer, Jos J. G. M van der Tol and André J. Boot
}

\begin{abstract}
In case of a large number of channels and a limited available optical bandwidth (limited laser tuning range) it may be necessary to chose a channel spacing near 0 or $11 \mathrm{GHz}$, where Rayleigh-and Brillouin backscattering introduce crosstalk in a bidirectional system. Although there is much literature on stimulated Brillouin scattering, that occurs at high powers, there is hardly any attention paid to what happens at relatively low input powers. Furthermore there are no system measurements which thoroughly investigate these effects.

We performed extensive bidirectional system experiments to investigate the crosstalk from Brillouin scattering in more detail, including its polarization properties. We found, that, while for high powers most of the energy is backscattered to a $11 \mathrm{GHz}$ lower frequency, for low powers there is no difference for the crosstalks at $11 \mathrm{GHz}$ higher or $11 \mathrm{GHz}$ lower than the signal frequency. The power budget is limited to $40 \mathrm{~dB}$ for both cases. The predicted $33.3 \%$ degree of polarization of low-power Brillouin scattering is experimentally confirmed for the first time.
\end{abstract}

\section{INTRODUCTION}

$\mathbf{F}$ BBER non-linearities, Stimulated Brillouin Scattering (SBS ) in particular, give an upper limit to the transmitted power [1]. A first type of limit occurs for high input powers exceeding the SBS-threshold, when most of the input power is scattered into an about $11 \mathrm{GHz}$ (at $\lambda=1550 \mathrm{~nm}$ ) frequency downshifted backward wave. This limit has been thoroughly investigated in literature. The SBS-threshold depends on the optical spectrum of the signal [2], [3] and can be improved (increased) by using a spread-spectrum scheme [4].

A second type of limit occurs in bidirectional systems, where the backward wave(s) can interfere with the signals in the opposite direction. It is mentioned in literature several times, that it is not wise to select a bidirectional channel spacing of $11 \mathrm{GHz}$, because of SBS (eg. [5]). However, in case of a large number of channels and a limited available optical bandwidth (limited laser tuning range) such a choice may be unavoidable. To our knowledge, there is only one reference ([6]), that contained a system measurement. It showed, that SBS can already introduce crosstalk at powers far below the SBS-threshold, but it did not systematically quantify the crosistalk. It is not know whether there is only crosstalk

Manuscript received October 18, 1993; revised November 29, 1993.

The authors are with Royal PTT Nederland N.V., PTT Research, P.O. Box 421, 2260 AK Leidschendam, Nederland. tel: $+31-70-3325484$, fax: +31-70 3326477, telex: $31236 \mathrm{ml} \mathrm{nl}$.

A. J. Boot is also with Eindhoven University of Technology, P.O. Box 513 , $5600 \mathrm{MB}$ Eindhoven, Nederland.

IEEE Log Number 9215905. from the higher frequency signal to the lower frequency counterpropagating signal or whether the crosstalk is mutual. Nor is it known whether the lower frequency experiences gain or whether the power of higher frequency signal will be depleted. Nor have the polarization properties of the crosstalk been studied in detail.

The purpose of this letter is to thoroughly investigate the crosstalk from Brillouin scattering (and also Rayleigh scattering) in a bidirectional system. Section II describes the experiments and presents the results. Section III gives a physical interpretation and discusses the implications of the results. Section IV gives the conclusions.

\section{EXPERIMENTS AND RESULTS}

The experimental set-up contains three DBR laser sources, an Erbium Doped Fiber Amplifier (EDFA), optical attenuators, an optical isolator, some couplers, some fiber-coil polarization controllers (POLC), a long piece of fiber (spool), a single balanced coherent receiver, and a ML93A optical power meter (PM). The DBR-sources are used as signal source (TX1), crosstalk source (TX2) and local oscillator, respectively. The signal laser is CPFSK (Continuous Phase Frequency Shift Keying) modulated at $155 \mathrm{Mbit} / \mathrm{s}$ using Manchester code and a $2^{10}-1$ pattern. The frequency deviation between mark and space is $395 \mathrm{MHz}$ (intermediate frequencies (IF): 345 and 740 $\mathrm{MHz}$ ). All lasers operate at $\lambda=1537 \mathrm{~nm}$ and have a linewidth of $2 \mathrm{MHz}$ FWHM. The EDFA is used to boost the power from the crosstalk source above $+10 \mathrm{dBm}$. The fiber length is $12.7 \mathrm{~km}$, the attenuation is $0.19 \mathrm{~dB} / \mathrm{km}$, the Brillouin shift is $10.88 \mathrm{GHz}$ and the Brillouin linewidth is $40 \mathrm{MHz}$. An optical isolator was put at the fiber input and all open fiber ends were terminated to eliminate optical reflections. Experiments are performed in three set-ups, see Fig. 1.

\section{A. Set-up 1: Bidirectional Crosstalk for Different Channel Spacings}

In the first set-up (Fig. 1a) the light of TX2 is injected in the backward direction through a 50/50 coupler to test the crosstalk from Brillouin-and Rayleigh scattering. First we measured BER-curves (Bit-Error-Rate). The sensitivity of our coherent receiver is $-54.7 \mathrm{dBm}$ at a BER of $10^{-9}$. Then the channel spacing was set at $0 \mathrm{GHz}$ and $\pm 10.9 \mathrm{GHz}$, respectively, where the crosstalk from Rayleigh and Brillouin scattering is at maximum. The channel spacing is positive, by 

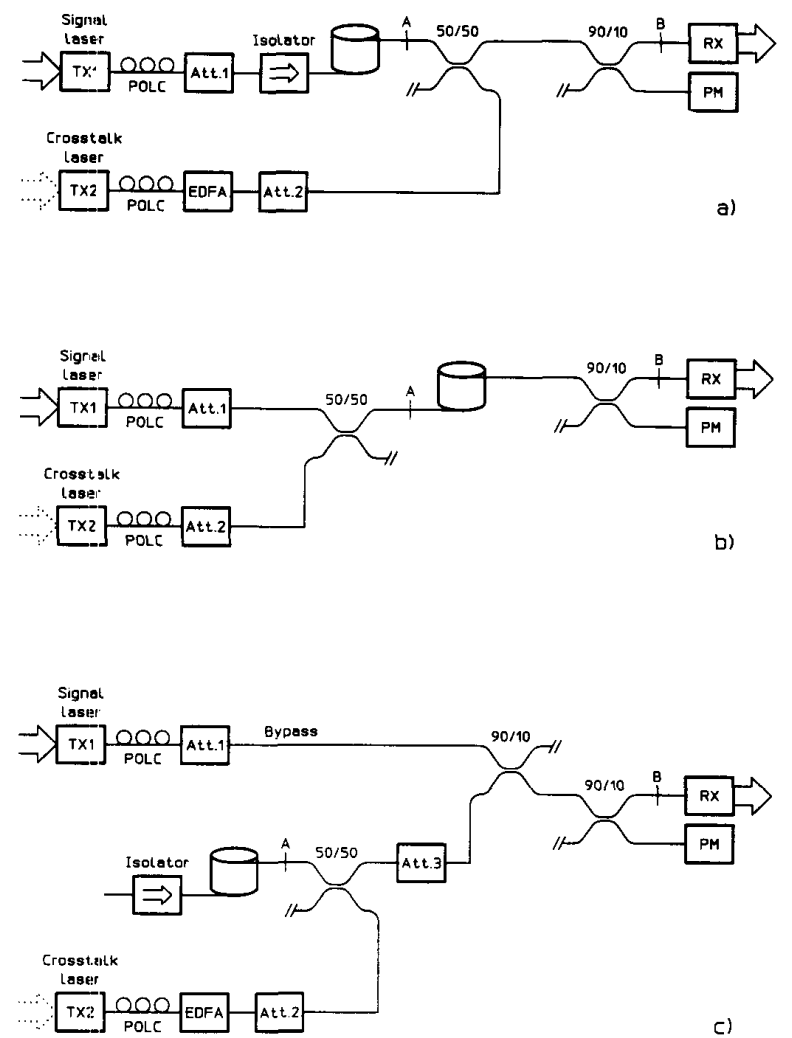

Fig. 1. Experimental set-ups. a) Set-up 1: TX2 light injected in backward direction b) Set-up 2: TX2 light injected in forward direction. c) Set-up 3: TX2 light injected in backward direction and TX1 light through bypass.

definition, when the optical frequency of the signal is larger than that of the crosstalk. The polarization controllers were adjusted manually to have optimum reception and maximum crossta]k. The crosstalk power, the total of the Rayleigh and Brillouin power, was measured at point $B$. This power is mainly the power of the Rayleigh scattering, as the Brillouin power was much lower. A crosstalk power of $-67.6,-48.0$, and $-47.8 \mathrm{dBm}$ was required to give a $3 \mathrm{~dB}$ penalty for 0 , -10.9 and $+10.9 \mathrm{GHz}$ respectively. These powers are -58.2 , -44.4 and $-43.9 \mathrm{dBm}$ to obtain a $6 \mathrm{~dB}$ penalty. So the crosstalk required to give a 3 (or 6 ) $\mathrm{dB}$ penalty is virtually the same for $+10.9 \mathrm{GHz}$ and $-10.9 \mathrm{GHz}$. All BER-curves have the same shape, there is no sign of a BER-floor.

Secondly we measured the power penalty for a BER of $10^{-6}$ at different crosstalk powers. The polarization was adjusted to determine maximum and minimum crosstalk. The channel spacing was set at 0 and $\pm 10.9 \mathrm{GHz}$. Since $+10.9 \mathrm{GHz}$ and $-10.9 \mathrm{GHz}$ gave identical results, only $+10.9 \mathrm{GHz}$ is presented, see Fig. 3. First the signal power is adjusted to $-57.5 \mathrm{dBm}$ for a BER of $10^{-6}$. Then a crosstalk power is injected, and the signal power is increased to give a BER of $10^{-6}$ again. We see, that for larger penalties, the penalty increases linearly with the input power. Apparently the crosstalk behaves as a linear additive noise source to the system noise. Polarization gives a $3 \mathrm{~dB}$ difference in input

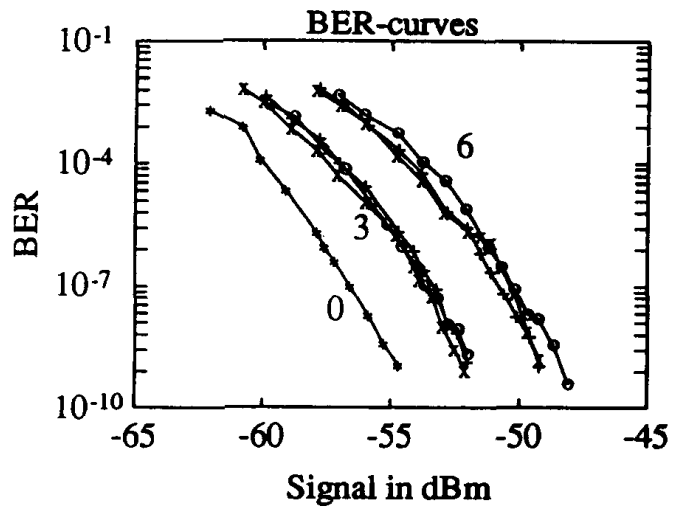

Fig. 2. BER-curves (set-up 1) for a 0,3 , and $6 \mathrm{~dB}$ penalty.*: no crosstalk, $0: 0 \mathrm{GHz}, \mathrm{x}:-10.9 \mathrm{GHz},+:+10.9 \mathrm{GHz}$, see text.

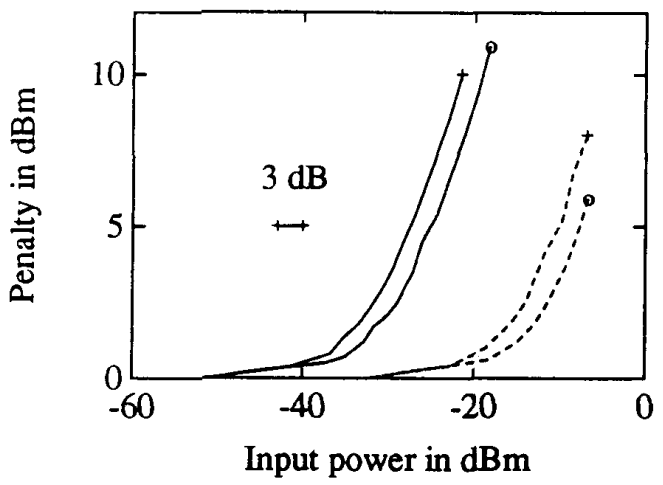

Fig. 3. Penalty versus input power (point $A$ ) for twopolarizations (set-up 1) Solid: $0.0 \mathrm{GHz}$ (Rayleigh), dotted: $+10.9 \mathrm{GHz}$ (Brillouin), +: identicalpolarization, $\mathrm{o}$ : orthogonal polarization.

power for a given penalty, both for Rayleigh—and Brillouin scattering.

Thirdly we measured the influence of the channel spacing, at a $3 \mathrm{~dB}$ penalty and a BER of $10^{-6}$ see Fig. 4ab. Three "dips" are visible, at which less crosstalk power can be tolerated. The central dip is from the Rayleigh backscattering, the other two dips are from the Brillouin scattering. The dips are bifurcated because of coherent imaging. We repeated this measurement for a modulated crosstalk laser and found only some slight differences in the fine-structure of the curves.

A BER of $10^{-6}$ and a $3 \mathrm{~dB}$ penalty were selected to make fast and accurate measurements. All results can easily be translated to other BER's and/or penalties using the results of Fig. 2 and 3.

\section{B. Set-up 2: Unidirectional Crosstalk (Reference Measurement)}

In the second set-up (Fig. 1b) the fiber and coupler change places and the light of TX2 is injected in the forward direction. The tolerable crosstalk is measured as a function of channel spacing, again for a BER of $10^{-6}$ and a $3 \mathrm{~dB}$ penalty, see Fig. $4 \mathrm{c}$. Now only the central dip is present. The tolerable crosstalk near $0 \mathrm{GHz}$ is about the same for Rayleigh backscattering (set-up 1) and direct crosstalk (set-up 2). Actually the direct 

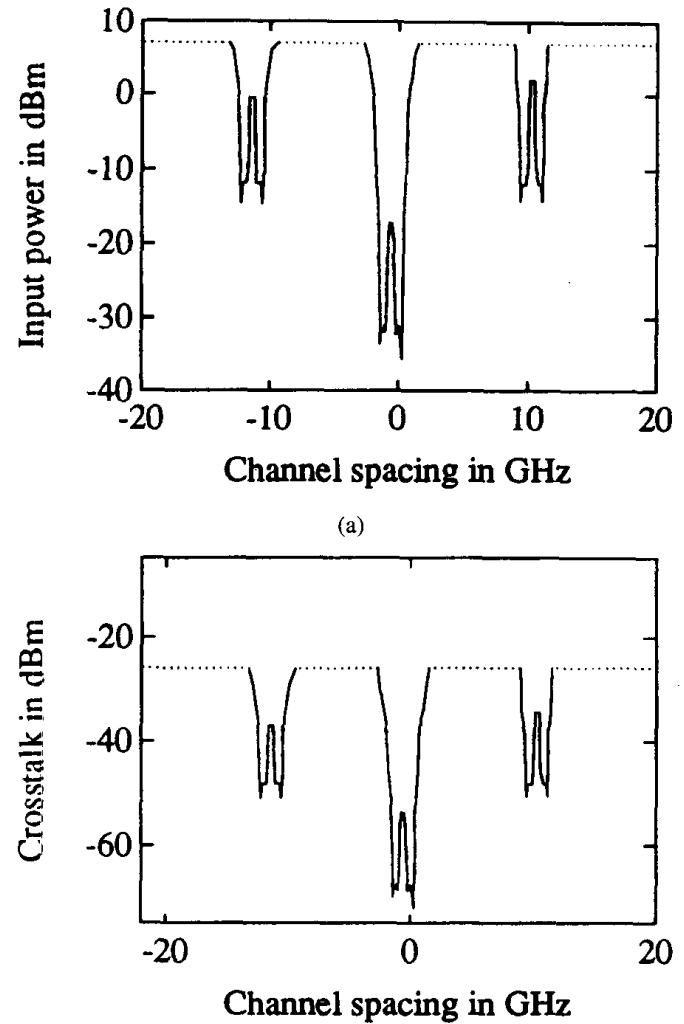

(b)

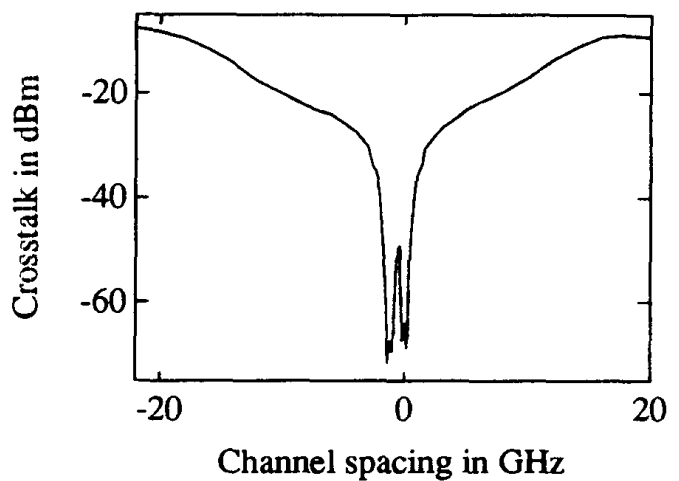

(c)

Fig. 4. Maximum allowable crosstalk as a function of channel spacing (BER $=10^{-6}$, penalty $\left.=3 \mathrm{~dB}\right)$, both bidirectionally $(\mathrm{a}, \mathrm{b}:$ set-up 1$)$ and unidirectionally (c: set-up 2). Vertical scale: a) TX2 power at point $A ; \mathrm{b}, \mathrm{c}$ ) the crosstalk power at point $B$.

crosstalk is $1.7 \mathrm{~dB}$ lower, because its degree of polarization is $100 \%$, while that of the Rayleigh backscattering is $33.3 \%$ [7].

\section{Set-up 3: Characterizing the Low-Power Brillouin Scattering}

In the third set-up (Fig. 1c) the fiber is bypassed and the coherent set-up is used to test the magnitude of the low--power Brillouin scattering. Here we make use of the unique properties of coherent detection: its high sensitivity and

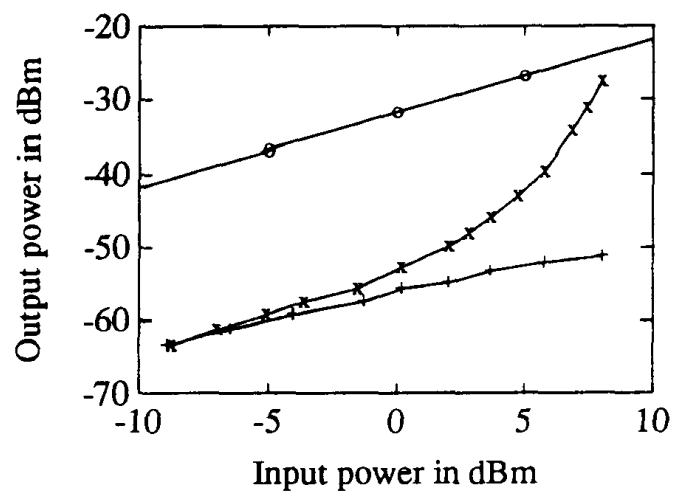

Fig. 5. Back scattered power as function of injected (crosstalk) power. Both scales refer to the optical powers at point $A$ (set-up 3). $0: 0 \mathrm{GHz}$ (Rayleigh), $\mathrm{x}:-10.9 \mathrm{GHz}$ (Brillouin), + : $+10.9 \mathrm{GHz}$ (Brillouin).

frequency selectivity. The signal of TX1 is kept constant, while the attenuators 2 and 3 are adjusted simultaneously to keep the crosstalk level constant at point $B$ (ie. $\mathrm{BER}=10^{-6}$, penalty $=3 \mathrm{~dB}$ ). The results are given in Fig. 5. For low input powers, the powers at -10.9 and $+10.9 \mathrm{GHz}$ are the same and increase linearly with the input power. For higher input powers, the power at $-10.9 \mathrm{GHz}$ increases exponentially, while the power at $+10.9 \mathrm{GHz}$ increases less than linearly.

\section{DISCUSSION}

The experiments clearly showed, that the crosstalk at -10.9 and $+10.9 \mathrm{GHz}$ are the same. At low power Brillouin scattering can be interpreted as reflection at thermally activated acoustical phonons. The reflected light experiences a Doppler shift of $-10.9 \mathrm{GHz}$ or $+10.9 \mathrm{GHz}$, depending on the direction of propagation of the phonons. As the phonons have no preferred direction of propagation along the fiber, the crosstalk powers from Brillouin scattering are the same ${ }^{1}$. At these low powers the energy tranfer between the channels (gain or depletion) is negligible. An asymmetry occurs for higher powers. Then the Brillouin process introduces gain at a $10.9 \mathrm{GHz}$ lower optical frequency, while the same process introduces depletion (attenuation) at a $10.9 \mathrm{GHz}$ higher optical frequency. This asymmetry can be seen in Fig. 5 .

The $3 \mathrm{~dB}$ difference for polarization, that was observed in Fig. 3. corresponds to a degree of polarization (DOP) of the scattered wave of $33.3 \%$. This can be explained from the properties of reflection [7], [8]: linearly polarized light is reflected with the same polarization, while circularly polarized light is reflected with the orthogonal polarization. Averaging over all polarizations, assuming random distributed retardations in the fibre, results in a DOP of $33.3 \%$. Here the predicted [8] $33.3 \%$ DOP of low-power Brillouin scattering is confirmed experimentally for the first time!

Although most experiments were made with an unmodulated crosstalk signal, the results are also valid with modulation. This is because we operate at optical powers far below

${ }^{1}$ In the more rigorous quantum-mechanical picture the identical dips at low power result from time reversal symmetry in the creation and annihilation of phonons 
the SBS-threshold. Fig. 5 showed that for these low powers Brillouin scattering is essentially a linear process. The optical spectrum of the backscattered signal is the incoming signal convolved with the small $(40 \mathrm{MHz})$ Brillouin linewidth [9]. So, while modulation increases the SBS-threshold because of the spectral broadening, there is little influence at low powers. This was experimentally confirmed.

Finally from Fig. 4 we can see the implication of selecting the channel spacing of $\pm 10.9 \mathrm{GHz}$. In that case the maximum transmitted power must be less than $-15 \mathrm{dBm}$ in a symmetrical bidirectional system, while it could be more than $+7 \mathrm{dBm}$ otherwise. As the receiver sensitivity is about -54 $\mathrm{dBm}$, the available powerbudget is then limited to $39 \mathrm{~dB}$. The powerbudget limitation is independent of the receiver sensitivity, as for a better receiver sensitivity less crosstalk can be allowed to have the same signal/crosstalk ratio at the receiver.

\section{CONCLUSION}

We made a thorough experimental investigation of the crosstalk from Brillouin (and Rayleigh) scattering in a bidirectional coherent system, including the polarization properties. The penalty was determined as a function of channel spacing and of the power in the reverse direction. The magnitude of the backscattered signal was measured.

When the channel spacing in a bidirectional system is selected to be $10.9 \mathrm{GHz}$ the available power budget is limited to $40 \mathrm{~dB}$. The crosstalk at $10.9 \mathrm{GHz}$ channel spacing is from low-power Brillouin scattering, where the stimulated Brillouin scattering is negligible. Therefore the crosstalk is the same for $+10.9 \mathrm{GHz}$ and $-10.9 \mathrm{GHz}$. Our measurements confirm the predicted $33.3 \%$ degree of polarization of the low-power Brillouin scattering for the first time.

\section{REFERENCES}

[1] Y. Aoki, K. Tajima and I. Mito, "Input power limits of single-mode optical fibers due to stimulated Brillouin scattering in optical communication systems," J. of Lightwave Technol., vol. 6, no. 5, pp. 710-719, 1988.

[2] T. Sugie, "Transmission limitations of CPFSK lightwave system due to stimulated Brillouin scattering in optical fiber,"J. of Lightwave Technol. vol. 9, no. 9, pp. 1145-1155, 1991.

[3] E. Lichtman, "Bit rate-distance product limitations due to fibre nonlinearities in multichannel coherent optical communication systems," Electron. Lett., vol. 27, no. 9, pp. 757-759, 1991.

[4] A. Hirose, Y. Takushima and T. Okoshi, "Suppression of stimulated brillouin scattering and Brillouin crosstalk by frequency-sweeping spread-spectrum scheme," J. of Optical Communications, vol. 12, no. 3, pp. 82-85, 1991.

[5] R. G. Waarts and R. P. Braun, "Crosstalk due to stimulated Brillouin scattering in monomode fibre," Electron. Lett., vol. 21 , no. 23, pp. 1114-1115, 1985.

[6] E. J. Bachus, R. P. Braun, W. Eutin, E. Grossmann, H. Foisel, K. Heimes and B. Strebel, "Coherent optical-fibre subscriber line," Electron. Lett., vol. 21, no. 25/26, pp. 1203-1205, 1985.

[7] M. O. van Deventer, "Polarization properties of Rayleigh backscattering in single-mode fibers," $J$. of Lightwave Technol, vol. 11, no. 12, pp. 1895-1899, 1993.

[8] M. O. van Deventer and A. J. Boot, "Polarization properties of stimulated Brillouin scattering in single-mode fibers," accepted for publication in J. of Lightwave Technol., 1993.

[9] E. Lichtman, R. G. Waarts and A. A. Friesem, "Stimulated Brillouin scattering exited by a modulated pump wave in single-mode fibers," $J$. of Lightwave Technol., vol. 7, no. 1, pp. 171-174, 1989. 